\title{
Cloud Theory and Fractal Application in Virtual Plants
}

\author{
Zhaohong Wang \\ College of Computer and communication engineering, Weifang University, Weifang, China \\ Email: wangzhhwfxy@163.com
}

\begin{abstract}
Plants is an important component of natural scene. Unfortunately, due to high level complexity of the structure of plant, simulating plant becomes extremely a difficult task. When the fractal theory is imported, it provides a broader development space for the plant modeling. With the development of the fractal research, virtual plant has become a hot and interesting research topic in computer graphics area. The virtual plants technology is very important in guiding the crop production, implementing the agriculture informationization and constructing the virtual environment. At present a single virtual plant modeling technology is quite mature, the method to generate a body of plants often uses the even algorithm or the normal algorithm, but a body of plants in the real world is not even, and is not normal also, the cloud model relaxes the precise determination membership function to expectation function with normal distributed membership degree, combines ambiguity and randomness organically to fit the real world objectively. So it has general applicability, producing a body of plants based on the cloud model can simulate plant's condition and the distribution well.
\end{abstract}

Index Terms-Virtual Plants; Cloud theory; Fractal; Expectations Function; Normal Distribution; Realism; IFS

\section{INTRODUCTION}

Virtualization is mapping the physical resources into logical resource, and then they can be managed conveniently without considering physical structures. In the future, all resources will transparently run in the virtual world across a variety of physical platforms, the resources management is fully automated distribution logically, and virtualization technology is the ideal tool to achieve the goal [1].

Computer with fractal theory of digital technology can create the patterns in which there are different kinds of objects whose styles seem to imitate the growth postures of plants coming from nature. We advanced some fractal algorithms, which make the algorithms performance more powerful and improve the efficiency of fractal images making, those strategies allow the art effect of fractal images to develop into be better and higher level and to exert their potentials. In order to be better to obtain rich and colorful fractal images, we introduced the basic principle of the cloud model of uncertainty intelligence used to imitate the drift phenomenon of plants growth in nature, producing different and surprising art patterns with different styles and colors. Furthermore, the images can be beautified by the computer image manipulation software, which let fractal images become design materials of ceramics patterns with great quality.

We can get visual, auditory, tactile, and other immersive experience in a virtual environment. Virtual plant is the simulation of plant using virtual technology through computer. Compared with traditional plant simulation models, the virtual plant model can research farmland, forests and complex ecosystem intuitively, found the law that traditional methods and technical means are difficult to observe; virtual plants in a virtual field experiment can partly replaced time-consuming, laborious, expensive tests, shortened the trial period of some research topics or save a lot of testing costs. Virtual plant is an integral part in virtual scene; it is a main method in developing some modern large computer programs [2].

Because of many uncertainties, there is much randomness in the simulation of plans, this has been considered in plant simulation algorithm. So a number of random factors have been acceded to the algorithms. However, these algorithms generally do not meet the requirements of fractal simulation; have yet to be further improved.

Cloud is a model described by linguistic values for representation of uncertain relationships between a specific qualitative concept and its quantitative expression. It is introduced to reflect the uncertainties of the concepts in natural languages. Cloud integrates the concept of randomness and fuzziness. The normal random number generation method in normal cloud generator algorithm overcomes the insufficiency of common method to generate random numbers. It can produce random numbers which can be predictable and replicated, and this random numbers present to be a random sequence as a whole, but do not show any obvious pattern, so it can meet the requirements of fractal simulation, thus combining cloud model and fractal to simulate nature plant is feasible. This Paper takes the plant simulation as an example to explore the feasibility of the combination of cloud model and fractal, and analyzes the uncertainty of the cloud fractal methods embodied in natural simulation. The results show that the method is effective.

\section{Generating Virtual Plant Methods}

Researchers began to simulate plant from the mid-60s of the 20th century. Established model can predict some 
complex index under different environmental conditions. Such models combined with Expert System have important guiding significance in completing scene modeling; guiding agriculture teaching and production. At present, plant simulation methods have three kinds as follow [3] [4].

\section{A. Fractal method}

Fractal method use plant fractal nature of the morphology (structural self-similarity) to produce graphics or images, this method include: L-System, IFS (iterated function system) method, DLA (diffusionlimited aggregation) model.

L-system describes the structure and growth of plants with formal language. It can describe the plant topology succinctly. But it is difficult to simulate the complex morphology of plants. IFS generate plant image using the initial plant images to iterate accordance with the known selection probability function. DLA method is: selecting a stationary particle in a flat grid as a seed then generates a particle farther away from the seed, let the particle walk around the directions of left and right, up and down along the grid randomly. It cohere to the seed if the particles collide with seed during walking, if the particles come to the border, it is absorbed by the border. Repeat the above steps, the image will be produced. Using DLA and its modified model can simulate some plant morphology, such as plant root growth process and seaweed morphology.

\section{B. Particle system}

The basic idea of particle systems is to use many tiny simple shapes as the basic element to represent the irregular nature of fuzzy scene, particle creation, disappearance and the trajectories are controlled by the shape of the object characteristics, creates the dynamic changes of scenery. A cylinder particles represent plant branches, a small spherical or small cubes of particles represent leaves, as long as combined these particles to form the model, we can achieve plant morphology simulation, in particle system, the random variables generate the necessary change in the decisive parameters. The drawback is scene expression involves a large number of basic primitives, so the design is a process of trial and modification, rendering the image must have a special shadow method.

\section{Three-dimensional structure model}

Three-dimensional structure model is based on the geometric description of the plant. It includes the static structure based on the geometric design and dynamic growth structures based on its own dynamic structure and adjust.

Geometric design method descried the geometric structure of plant with a set of geometric values and the description rules, so to establish the three-dimensional structure model of the plant. The model based on the plant-specific branching patterns, we use branch angle, branch length ratio, attractor geometry, to implement the control of the plant geometry changes. This method can set up various forms of plant structure quickly and intuitively.

Growth method gets the plant morphology by simulating the plant growth and development. Different growth control methods will produce different simulation algorithms, and some control means is related to the plant their own structure only, some take "virtual plant hormones" as a means of regulating the growth method. Growth method has potential at simulating the plant growth, suitable to simulate plant in the botany, the physiology and the ecology.

These studies are focused on a single plant simulating method, in order to simulate farmland, or a body of plant in a virtual environment, we need generating algorithms based on a template plant, the simplest way is to use random algorithm or uniform algorithms to generate them, or based on the known probability function to generate them, of course, better algorithms are based the normal distribution function.

Many of the probability distribution of random variables can be described approximately by a normal distribution to in production and scientific experiments. For example, under the same conditions of production, product strength, compressive strength, diameter, length and other indicators; the lengthens of the same organism, the weight and other indicators; the same seed weights; measurement error of the same object; the impact point of deviation along the a direction; the annual precipitation of an area, and so on. In general, if an amount is affected by many tiny independent random factors, it will be supposed that the amount's distribution is normal. In theory, the normal distribution has many good properties, many probability distribution can be approximated by normal distribution; there are some commonly used probability distribution derived from it directly.

Normal distribution is the extremity of many important distributions; normal distribution density function and distribution function have a variety of good nature and relatively simple mathematical form, so they are widely used in theory and practice. But many random phenomena cannot be described by a strict normal distribution. If the factors determined the random phenomenon have a certain degree of interdependence, or when they affected the random phenomenon alone, is not even and small, they do not constitute a normal distribution also, can only be replace by normal distribution approximately.

Probability theories deal such situations with joint distribution, but joint distribution usually is very complex and difficult to practice. Cloud models do not use accurately membership function to describe problem, but by constructing the expectations function of normal membership distribution to simulate the real world normal distribution, it has a relatively simple mathematical form, can simulate the similar normal distribution well, has a very good fit for the real world plant distribution and growth which have randomness and ambiguity, it can simulate the real world the real situation better. 
When a body of plants is simulated, if we use a known probability function, or use a completely random function, the virtual reality is not so high, because the reality of the plants does not fit with the known probability function to change, nor is it completely random grow. In nature, most objects are not ordered, stable, and certain, but they are in a chaotic, unstable and non-equilibrium random state. There are no two plants grow completely in the same way. Even with a same kind of plant, their shapes are also different, such as stem height, flower position, seed shape, and so on. In particular the environment caused the morphological variation. For example, grain crops have the larger ears and more seeds for adequate fertilizer. For this, the effect of the plants simulation using of the normal or the random methods are obviously stiff, not so vivid. If the main features of the plant are retained, the differences of the plant's details are wanted, in order to generate a body of more vivid and realistic plants, cloud model should be introduced. Cloud model combines ambiguity and randomness organically based on the statistics science and fuzzy mathematics to study the universal law of uncertainty in human society. Use cloud model to simulate the plant's self-similarity, disorder, instability, non-equilibrium and Randomness have higher accuracy than the other method.

When the random phenomenon are described by cloud model, the normal distribution is expanded to similar normal distribution, with a new independent parameter Super entropy, to measure the degree of deviation from normal distribution, this approach is more widely used than the simply normal distribution, is easier than the joint distribution, easy to represent and manipulate. Here's what the cloud which can generate the similar normal distribution [5] [6].

\section{CLOUD MODEL}

The digital features of the normal cloud characterized by three values with the expectation Ex, entropy En excess entropy He) fig.1.

subordination

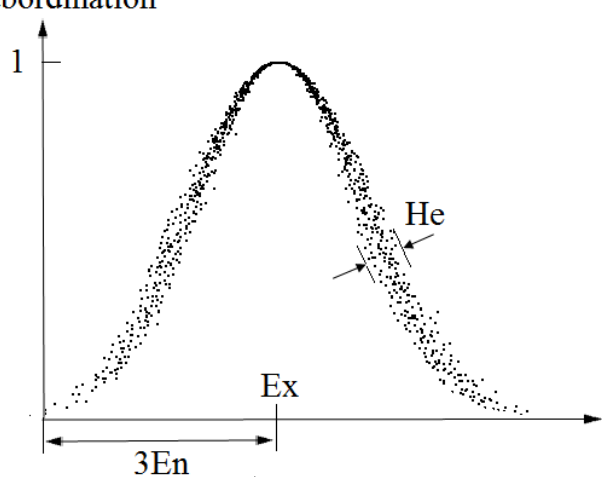

Figure 1. The digital features of the normal cloud

The expected value Ex: Ex is the center value of the concept domain and the most representative qualitative value of the concept, it should be $100 \%$ belongs to the concept; Ex reflect the value of the information center of the qualitative knowledge.

Entropy En: is a qualitative measure of the concept's fuzziness, reflecting the accepted range values of the concept domain; the greater the En is, the range of values represented by the concept is the greater, the more vague the concept is.

Hyper entropy He: can be described as entropy En of entropy, reflecting the degree of dispersion of the cloud droplets.

Since entropy reflects the range of the domain language can be the accepted by people, and hyper entropy $\mathrm{He}$ is entropy's entropy, $\mathrm{He}$ is small, random entropy En change is small, indicating that people is more consistent that a qualitative value of belong to the concept; on the contrary, if $\mathrm{He}$ is large, randomized entropy En change a lot, that indicates people have big differences in understanding a qualitative value belong to the concept, which reflects the understanding is not the same whether the qualitative concepts is accepted. As a result, people do not know the extent to which a cloud droplet belongs to the concept. So in order to reflect the concept that people have some same understanding, while there are some differences, it is more appropriate that En and He ratio is usually between 15 to 40 . Fig. 2 (a-f) He changes reflects the different situations.

We can be seen Fig. 2 (f), although En equal to1, but $\mathrm{He}$ is 50 , this indicated that the random entropy can be broadly range from -149 to 151 to calculate the degree of membership of each cloud droplets, so that the changes of random entropy will be very large, almost like that each cloud droplet will use a completely different entropy [5].

Thus, use the cloud model to represent the membership function is to determine the digital features of the cloud model, if the concept itself is clear and it is understood consistently by almost all people, you can use smaller En and He. If the concept itself is vague, our understanding is very inconsistent, you can use larger En and He.

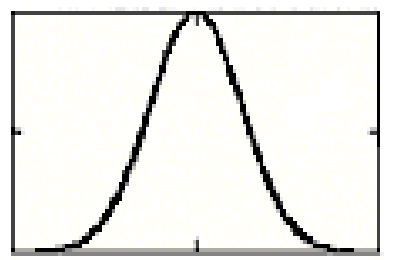

(a) $E x=0$ En=1 $\mathrm{He}=0.01$

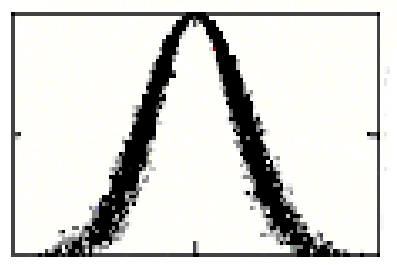

(c) $\mathrm{Ex}=0 \mathrm{En}=1 \mathrm{He}=0.1$

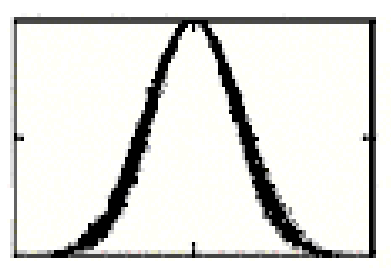

(b) $\mathrm{Ez}=0 \mathrm{En}=1 \mathrm{He}=0.05$

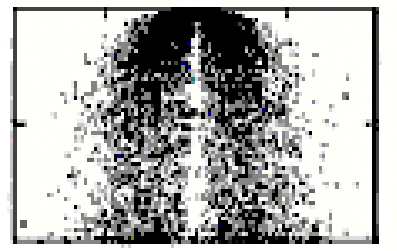

(d) $\mathrm{Ex}=0 \quad \mathrm{En}=1 \mathrm{He}=1$

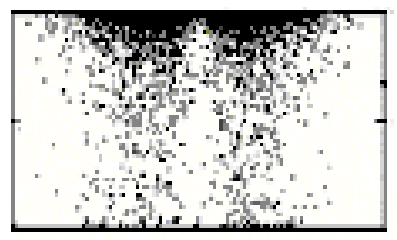

(e) $\mathrm{Ex}=0 \quad \mathrm{En}=1 \mathrm{He}=10$

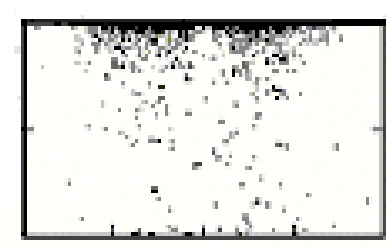

(f) $\mathrm{Ez}=0 \mathrm{En}=1 \mathrm{He}=50$

Figure 2. Cloud model changes with $\mathrm{He}$ 
The normal cloud is the most important cloud model, because various branches of the social and natural sciences have proved the normal distribution's universality. The equation of normal cloud curve:

$$
\operatorname{MEC} \mathrm{A}(\mu)=\mathrm{e}^{-\frac{(\mu-\mathrm{Ex})^{2}}{2 \mathrm{En}^{2}}}
$$

Definition 1: $\triangle X$, a variable of any inter-district of basic variable $\mathrm{X}$, who's Contribution to qualitative concept $\tilde{\mathrm{A}}$ is $\triangle \mathrm{C}$ :

$$
\Delta \mathrm{C} \approx \mu_{\tilde{A}}(\mathrm{x}) * \Delta \mathrm{x} / \sqrt{2 \pi} \mathrm{En}
$$

Obviously, all the elements total contribution $\mathrm{C}$ to the conceptual domain $\tilde{\mathrm{A}}$ is:

$$
\begin{aligned}
& \mathrm{C}=\frac{\int_{-\infty}^{+\infty} \mu_{\tilde{\AA}(\mathrm{x}) d x}}{\sqrt{2 \pi} E n}=\frac{\int_{-\infty}^{+\infty} e^{-(x-E x)^{2} / 2 E n^{2}} d x}{\sqrt{2 \pi} E n}=1 \\
& \mathrm{C}=\frac{1}{\sqrt{2 \pi} E n} \int_{E x-3 E n}^{E x+3 E n} \boldsymbol{\mu}_{\tilde{A}}(x) d x=99.74 \%
\end{aligned}
$$

for a qualitative knowledge, clearly, the expected curve is a normal curve. Elements do not fall into [Ex-3En, Ex-3En] can be ignored, because it has been proved that approximately $99.74 \%$ elements of model fall into the range of [Ex-3En, Ex-3En] by the mathematical characteristics of normal distribution.

\section{A. Cloud Generator}

Cloud generation algorithms can be achieved using the software, and also can be implemented by firmware, algorithms generated cloud droplets by the three digital characteristics of cloud are called Cloud Generator, also known as the basic cloud generator, it is the basis for uncertainty reasoning, it produces a cloud droplets which show some forms distribution[9][10][11]. Fig.3 Schematic diagram of a forward cloud generator.

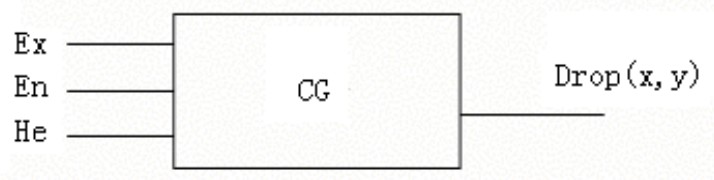

Figure 3. The generator of the forward cloud model

Algorithm 1: forward cloud generator algorithm

/ * Input: the expectations of the cloud forecast model Ex, entropy En and hyper entropy He

Output: predicted values $\mathrm{X}=(\mathrm{x} 1, \mathrm{x} 2 \ldots \mathrm{xi} \ldots, \mathrm{xn}) * /$ \{

(1) Produce normal random entropy En' with expectation En, MSE He;

(2) produce a normal random number $\mathrm{x}$ with expectation Ex, MSE E n';

(3) Calculate $y=e^{-\frac{(x-E x)^{2}}{2 E n^{2}}}$

(4) Take (x, y) as a forecast data;
(5) Repeat steps 1 through 4, until a required number of forecasts data produced.

\}

The step 2 in forward cloud generator algorithm, $99.74 \%$ of the forecast data will fall (Ex-3En, Ex $+3 E n)$ range from the statistical knowledge; so that you can predict the precise degree of reality to fit the data well. The predictive value of $y$ is essentially the membership degree of $\mathrm{x}$ under this model, it is just a reference value, if the actual prediction system does not require, dispense it, of course it can be used for membership degree analysis.

Random number generation method is the key to the whole algorithm. Almost all programming languages have a function that generated uniform random number between [0,1], according central limit theorem [12][13], the way generate normal random number using uniform random function is as follows:

Algorithm 2: Algorithm for generating normal random numbers

/* Input: Ex, En

Output: the random number $\mathrm{x}$ in accordance with the normal distribution $\mathrm{N}(\mathrm{Ex}, \mathrm{En}) * /$

\{

(1) Generate 6 uniform random number between $[0,1]$, calculate the sum of them: $\operatorname{sum}=\sum_{i=1}^{6}$ rand $_{i}$

/ / Randi is uniform random number between [0,1]

(2) generate random number $\mathrm{x}$ with the standard normal distribution $\mathrm{N}(0,1)$ according to the central limit theorem:

$$
\mathrm{x}=\text { sum- } 6
$$

(3) Transform $x$ to meet the normal distribution $N(E x$, En)

$\mathrm{x}: \mathrm{x}=\mathrm{Ex}+\mathrm{x}^{*} \mathrm{En}$

\section{(4) Return $x$}

\}

Cloud can be generated according to different conditions, given the value of $\mathrm{x}$ in the domain are called $\mathrm{X}$ condition cloud generator (see Fig.5), given the degree membership a particular value are called $\mathrm{Y}$ condition cloud generator (see Fig.6 b). $\mathrm{X}$ condition cloud generator generated cloud droplets in a same vertical line, the abscissa values are $\mathrm{x}$, the vertical axis were the probability distribution of membership values. $Y$ condition cloud generator generated cloud droplets in the same horizontal line, and divided into two groups by the expected Ex, the membership value of the vertical axis are $\mu$, the two groups of the abscissa values were, respectively, the probability distributed. The two conditions cloud generator models are basics of uncertainty reasoning. 
Currently, the research and the application about Cloud model theory has achieved some success, for example: The cloud model is used in intelligent control, successfully achieved the transform of real-time balancing posture of three levels inverted pendulum; in data mining, achieved the knowledge discovery of association rules; used to simulate the evolution and the fractal of the nature phenomenon, which explains the 24 solar terms. It generated hopping sequence code for hopping frequency radio, it also used in the evaluation for large system performance and other fields. The combination of random and fuzzy organically, make it has unique advantages in the simulation of natural phenomena. We believe the cloud model is a powerful tool for knowledge representation and qualitative and quantitative transform; it will be widely used in more areas [10].

Cloud is a model described by linguistic values for representation of uncertain relationships between a specific qualitative concept and its quantitative expression. It is introduced to reflect the uncertainties of the concepts in natural languages. Cloud integrates the concept of randomness and fuzziness. The normal random number generation method in normal cloud generator algorithm overcomes the insufficiency of common method to generate random numbers. It can produce random numbers which can be predictable and replicated, and this random numbers present to be a random sequence as a whole, but do not show any obvious pattern, so it can meet the requirements of fractal simulation, thus combining cloud model and fractal to simulate nature is feasible [14]. This Paper takes the plant as an example to explore the feasibility of the cloud model application, and analyzes the uncertainty of the cloud methods embodied in natural simulation.

\section{COMPUTER SIMULATION OF A BODY OF PLANTS BASED ON CLOUD MODEL FRACTAL}

The self-similarity is the basic nature of the fractal. And it supplies a theoretical basis for the computer simulation of fractal graphs. In nature, among the nonlinear complex phenomena there exists some self similar or scale. Flee features, which are simple but important. Using fractal theory to depict those natures scenery is feasible. Because it can recur the original object through only a small amount of information. And it has many good features: less specific information, easiness and high accuracy. However, the natural objects are not strictly self-similar. Their self-similarity in the statistics is proved true and the structure of self-similar level is limited. Therefore, in the fractal simulation algorithm, we can add a random value within a certain scope to each parameter which controls fractal to reflect the randomness. Therefore, in order to achieve a more realistic simulation of nature, how to produce random number to meet the requirements of fractal simulation is one of the key factors of the fractal process.

For the above parameters of cloud model, we can use it to simulate the natural phenomena such as a body of plant, take the basis plant model as the resource, get the height of plants, plant spacing, and deviation from the vertical direct angle, color, etc. set the mean values of above parameters as a cloud model expectations Ex, to definite the cloud model entropy and hyper entropy depending on the circumstances, if the plants and management are good such as farmland, it can give a smaller entropy and super entropy, so the plants grow even, if the farmland management was worse, you can give medium-sized entropy and hyper entropy, to reflect the growth of plants is not very even, if in the wild environment, we have to give greater entropy and hyper entropy, to reflect a very random and fuzzy plant growth phenomena, Fractal tree is show in fig. 4 .

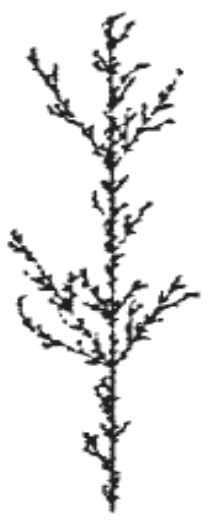

Figure 4. Fractal tree

Generated a body of plants using this model, even if the three figures of the cloud model are same, the algorithm run at different times will generate a different body of plants, represent the natural randomness phenomena, but the change are not so severe to affect our understanding of the phenomenon, so the method can simulate natural scenes vividly.

Simulation algorithm is given below.

Algorithm 3: Simulation Algorithm

/* Input: plant model, the mean values of the plant height, plant spacing, the angle of deviation from vertical, the color of the model and the cloud model entropy En and hyper entropy $\mathrm{He}$

Output: the simulation of the body of plant based on the cloud model */

\{

(1) Take the mean values of plant height, plant spacing, the angle of deviation from the vertical direction, the color as the clouds model expectations Exi;

(2) Generated normal random entropy Eni based on the expected value Eni, the mean square Hei;

(3) Generated normal random number Xi based on the expected value the Exi, mean square Eni;

(4) Take Xi as a plant model and draw the plant;

(5) Repeat steps 1 through 4, until all plants generated.

\}

Using this algorithm with the same plant model, only change En and He of the method created the simulation scenarios are as follows: 
For more uniform plants, we can take the original model parameters as the algorithm Ex, En and He take smaller values, and we generate more uniform plants in fig.5.

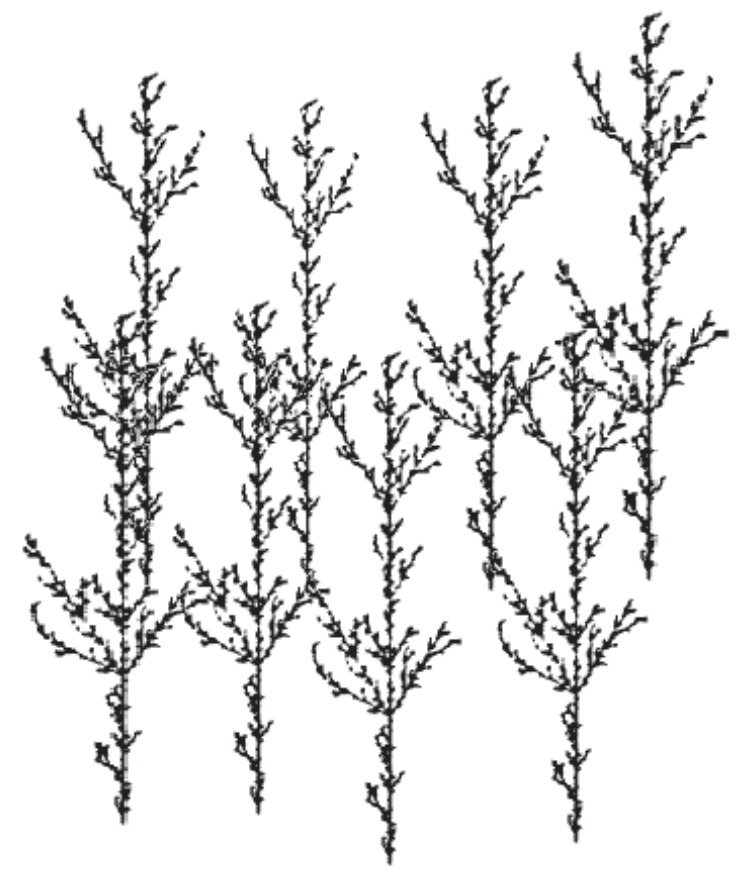

Figure 5. The more even plants generation

En and He are 0.2 and 0.01. En selection can consider the nature of the normal distribution, that is about $99.74 \%$ in all values with the same distribution of the $\mathrm{x}$ fall into the range [Ex-3En Ex $+3 E n]$, we can approximately take the Ex-3En as a probability distribution $X$ minimum, $\mathrm{Ex}+3 \mathrm{En}$ as the maximum value of the probability distribution of $\mathrm{X}, \mathrm{En}$ is one-sixth of the difference between Xmax and Xmin, so to further improve the algorithm's Adaptability.

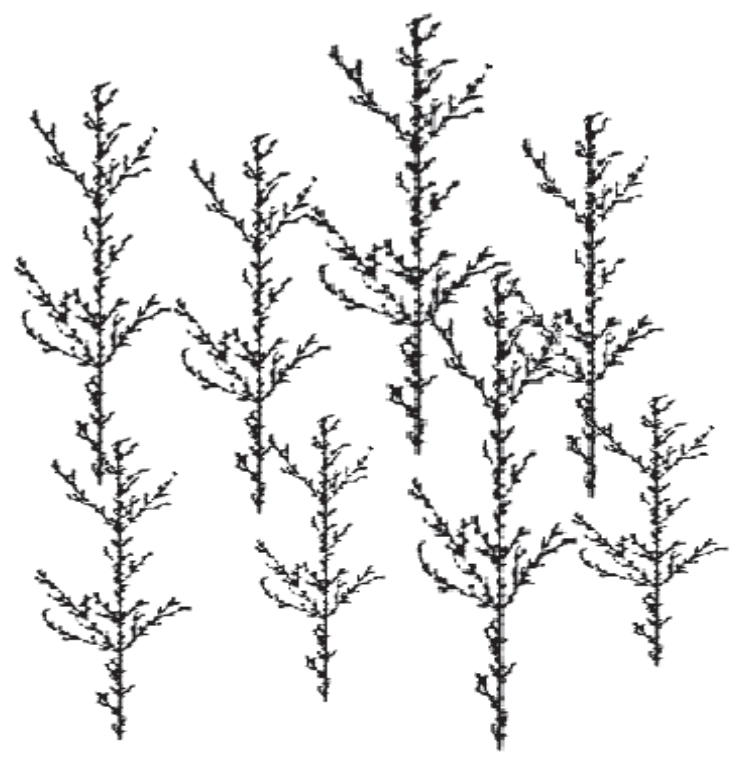

Figure 6. The more uneven plants generation
The uniform plants generated in fig.6, the algorithm's En and He take 0.4 and 0.08 . Of course, we can continue to let these two parameters become larger, and get the plants which distribute more unevenly.

\section{CONCLUSION}

In the past 30 years, the plants modeling, simulation, visualization and application have developed quickly. But when many models and algorithms simulated real natural phenomenon, they either are static or continuously adjust the complex parameters. Use tested fractal plant model, with the universality and simplicity cloud model to simulate the nature plant, the plants distribution is similar normal distribution, but affected by other kinds of random phenomenon they own uncertainty themselves. We developed a dynamic plant simulation algorithm, the algorithm are wildly used in virtual fields, virtual scene.

Integrating cloud model and fractal, we get cloud fractal model, and extended cloud is realized in it. Cloud model has the advantage to analyze fuzziness and randomness synthetically and fractal theory has the advantage to analyze phenomenon of self-similar and scale-free, the combination of the two methods is all effective way to analyze uncertainty problem. In this paper the cloud fractal has been applied to plants simulation, it shows that this cloud fractal method is effective.

\section{REFERENCES}

[1] Zhang Maojun Virtual reality system, Beijing: Science Press, in chinese, 2002

[2] Xiang Kai, VRML-based network class virtual research and Implement, Computer Systems Applications, in chinese, 2007, (3) pp. 105-107

[3] $\mathrm{Hu}$ Baogang, Zhao Xing, Plant growth modeling and visualization-Review and Prospect , Automation Technology, in chinese, 2001, 27 (6) pp. 816-835

[4] Wang Hai, Lin Shan, Huang Xinyuan, Evaluation and comparison of plant generation software, Computer Simulation, in chinese, 2006, 23 (8) : pp. 177-180

[5] Liu Chang-Yu, Li Deyi, Du Yi, Statistical analysis of normal cloud model, Information and Control, in chinese, 2005, 34 (2), pp. 236-239

[6] Li De-yi and Liu Chang-yu, Discussion of the universal nature on the normal cloud model. China Engineering Science, in chinese, 2004, vol. 6, pp. 28-33

[7] Li Deyi, Meng Haijun, Shi Xuemei, Membership cloud and membership cloud generator, Computer Research and Development, in chinese, 1995, 32 (6) pp. 15-20

[8] Li Deyi. Triple inverted pendulum control and dynamic balance based on clouds model, China Engineering Science, in chinese, 1999, 1 (2), pp. 41-46

[9] Li Deyi, Du Yi, Uncertainty Artificial Intelligence, Beijing: National Defense Industry Press, 2005

[10] Lu Huijun, Wang Ye, Li Deyi, Reverse cloud application in the qualitative evaluation, Journal of Computers, 2003, 26 (8) pp. 1009-1014

[11] Zhu Xiaohua, Wang Jian. Conditions and prospect for the application of fractal theory in geography. Exploration of Nature. 1999, 18(3): 42-46 
[12] Huang tian-yun,Zhang chuan-wu.On use of fractal interpolation algorithm to generate natural scenery[J].Computer engineering and design, 2007, 28(16): 3994-3997.

[13] Ruan kun, Fan yin, Li hui-jun, etc. Modeling cloud based on midpoint deflection algorithm[J].Journal of PLA university of science and technology,2003,4(1):99-102.

[14] Shuigen Li, "Fractal Theory", Higher Education Press, 2004.

[15] Jizhong Zhang, "Fractal Theory", Tsinghua University Press, 2001.

[16] Bowen Sun, "Fractal Algorithm and Programming", Science Press, 2004.

[17] Reeves W T, Blau R. Approximate and probabilististic algorithms for shading and rending structured particle systems. Computer Graphics, 1 985, 1 9(3):313. 322

[18] Honda H. Description of the form of trees by the parameters of the tree-likebody: Effects of the branching angle and the branch length on the shape of the tree-like body. Journal of Theoretical Biology, 1971,3 1:33 1-338

[19] Honda H, Fisher J B. Ratio of tree branch lengths: The equitable distribution of leaf clusters on branches. Proceedings of the National Academy of Sciences USA, 1979, 76(8):3875-3879

[20] Honda H, Tomlinson P B, Fisher J B. Computer simulation of branch interaction and regulation by unequal flow rates in botanical trees. American Journal of Botany, 1981, 68:569-585

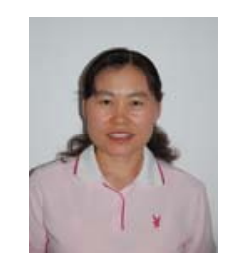

Zhaohong Wang was born in China in 1970. She earned B.S. degree in the field of management information system in 1993 from Jilin University of Technology, Changchun, P.R.China, and earned M.S. degree in the field of Computer Application Technology in 2004 from Shandong University of Science and Technology, Taian, P.R.China.

She is an Associate Professor of College of Computer and communication engineering, Weifang University, Her research was in the area of knowledge management, data mining, and intelligent algorithm, and her research interests have led to a number of publications. She is always looking forward to collaborations from similar ambitions. 